\title{
Electric Vehicle Energy Consumption Estimation for a Fleet Management System
}

\author{
Abbas Fotouhi ${ }^{1 *}$, Neda Shateri ${ }^{2}$, Dina Shona Laila ${ }^{2}$, Daniel J. Auger ${ }^{1}$ \\ ${ }^{1}$ Advanced Vehicle Engineering Centre, School of Aerospace, Transport and Manufacturing (SATM) \\ Cranfield University, Bedfordshire, MK43 OAL, UK \\ ${ }^{2}$ School of Mechanical, Aerospace and Automotive Engineering, Coventry University, Priory Street, \\ Coventry, CV1 5FB, UK \\ * Corresponding author: Abbas Fotouhi, email: a.fotouhi@cranfield.ac.uk
}

\begin{abstract}
Accurate estimation of vehicles' energy consumption is a demanding task. It might not be so critical for conventional vehicles because of their high travel range however, this is something important for electric vehicles (EVs). On the other hand, EVs with less energy on board, need more accurate energy management systems. This study focuses on the development of an energy consumption estimation model to be used in an EV fleet management system (FMS). The proposed estimator consists of a vehicle model, a driver model, and terrain models. It is demonstrated that a combination of these three parts can provide an accurate estimation of EV energy consumption on a particular route. As part of this study, a commercially-available passenger car is modelled using MATLAB/Simulink. A number of specific routes are selected for EV road testing to be driven for simulation model verification. In the second part of this study, the impact of energy consumption estimation accuracy is investigated at a larger scale for a fleet of EVs. It is quantitatively demonstrated how much sensitive is the performance of a FMS to the accuracy of the energy estimator. Simulation results have shown that the total energy consumption of an EV fleet is decreased significantly by improving the estimation accuracy. It is also demonstrated how the uncertainties in EV energy consumption estimation limits the overall performance of a FMS.
\end{abstract}

Keywords: fleet management system, electric vehicle, energy consumption estimation, modelling and simulation, experimental test. 


\section{Introduction}

The number of electric vehicles (EVs) is increasing quickly and with new regulations on clean and sustainable transportation systems, this trend would continue. More use of EVs makes new opportunities and also new challenges. Private EV users have different expectations and usage patterns while they still have some kind of range anxiety. "Accurate" estimation of EVs range is a demanding task that depends on a number of uncertainties such as variations in weigh, road and weather conditions, driving and traffic conditions, and degradation or change in components, etc. In addition to private vehicles, fleets of taxis or commercial delivery vans and trucks can play a significant role in the transportation sector. Since the next generation of fleets are likely to be electrified, new strategies are needed for proper management of EV fleets energy demand and charging. Even for scheduling of the fleet, new algorithms are necessary in which the range of EVs and the charging time are considered. At the present time, a scheduling fleet management software does not have to consider range limits in conventional non-electric vehicles nor the fuelling time, as refuelling is quick, and easily managed by the driver. In addition, all the existing fleet management algorithms are not necessarily optimal in terms of energy consumption. An EV fleet can be used optimally in a way to minimise the total fleet energy consumption to do a certain task. Summarising the above discussion, an important part of an EV fleet management system (FMS) or a private EV trip planner is "energy consumption prediction" which is the topic of this research.

Relevant literature can be categorized into two main groups: (i) studies which are focused on FMS and vehicle routing, and (ii) studies in which vehicle energy consumption is investigated.

There are a number of studies in the literature where the vehicle routing problem (VRP) and trip-to-vehicle assignment problem are investigated for conventional vehicle fleets. The VRP that was originally introduced by Dantzig et al. (1959), is a more general form of the traveling salesman problem (TSP) adjusting for customers' demands and vehicles' capacities as discussed by Giglio et al. (2004). The TSP is a nondeterministic problem that is defined as a salesman who wants to visit a number of cities only once, starting from any of them but returning to the same point at the end (Lin, 1965). The goal is to find the shortest route among all possible routes. For many years, efforts have been done to solve TSP using a wide range of methods. The VRP is an extension of the TSP in which a vehicle should visit a number of destinations and then returns to the original location. 
Considering the vehicles routing and scheduling as the main function of a FMS, there are two possible scenarios here: off line and online routing and scheduling. The same classification is presented by Betz et al. (2016) using two other terminologies: static and dynamic scheduling. In off line (static) scheduling, all trips are identified and planed at the beginning of the day without any changes during the course of the trip, whereas in online (dynamic) scheduling, there is a flexibility to change the plan in real-time. This flexibility is quite useful when a breakdown or an accident happens. There are many FMS algorithms available in literature; for example, the algorithms developed to solve the standard TSP and its variations, including Genetic Algorithm (GA), Simulated Annealing (SA) (Kirkpatrick et al. 2007), Tabu Search (Cordeau et al. 2001) and Ant Colony Optimisation (ACO) algorithms (Narasimha et al. 2011).

Although there are various studies in literature focusing on VRP and FMS for conventional vehicles (Bandeira et al. 2016), not enough work have been conducted specifically for EV fleets. In a study by Betz et al. (2016), a mixed fleet of conventional vehicles and EVs was investigated. As a result, a new model was proposed to analyse the financial and ecological effects of substituting conventional vehicles with EVs and to provide a personalized offer for the optimal fleet composition based on the number of trips and vehicle specifications. In a study by $\mathrm{Hu}$ et al. (2016), a review of the optimization and control techniques of smart EV fleet charging is presented and the services that can be provided by a fleet operator to other actors in a smart grid are discussed. In a study by Chao et al. (2013), differences of fleets of electric buses and conventional diesel buses are investigated. It is demonstrated that such differences creates the need for great changes in vehicle scheduling methods.

One of the key elements in a FMS is to know the distance between each point in the target area where the fleet operates. Simply speaking, the vehicle routing algorithm should know the distance between point $\mathrm{A}$ and point $\mathrm{B}$ when dispatching the vehicle from $\mathrm{A}$ to $\mathrm{B}$. This is even more critical when talking about a fleet of EVs since the range of an EV is much less than a conventional vehicle. To be more accurate, the "distance" should be replaced by "energy" that is needed to go from A to B. The energy consumption generally depends on three groups of parameters: 1) the vehicle specifications, 2) terrain characteristics and 3) driving style and traffic condition. In the next paragraphs, some earlier studies in which vehicle energy consumption has been investigated, are reviewed.

As stated by Jayakumar et al. (2014), accurate estimation of vehicle energy consumption is a demanding task because of the effects of different factors such as driving style, wind speed, 
traffic condition and uncertainties in the vehicle model. In that work, the effect of temperature on aerodynamic drag, rolling resistance and mechanical system efficiencies were also investigated. In a study by Jimenez et al. (2015), EV energy consumption estimation is investigated with focus on driving style. A neural network was trained using driver and traffic data to estimate the energy consumption. According to the results presented by Jimenez et al. (2015), driving style and driver specifications can cause up to $25 \%$ difference in EV energy consumption. In a study by Vatanparvar et al. (2018), the effect of driving style on EV energy consumption was investigated. The driving behaviour was analysed using artificial neural networks and nonlinear auto-regressive methods to build a driving behaviour model.

In a study by Trigui et al. (2017), EV energy consumption was estimated based on distance, vehicle mass and rolling resistance force. The vehicle mass and the rolling resistance coefficient were estimated in real-time based on longitudinal vehicle dynamics using a recursive least squares algorithm. For this purpose, the relationship between the electrical power and the actual mechanical power was formulated. A neural network structure was also developed to estimate vehicle mass and rolling resistance coefficient by Trigui et al. (2016). Vehicle mass estimation for EV energy estimation and trip planning is extended by Maalej et al. (2015). In a study by Adam et al. (2012), an algorithm for estimation of the energy consumption of an EV along a predefined route is proposed. A number of stopping points from a digital map is provided by the route course including longitude, latitude and altitude. An optimized spline is used to interpolate the two-dimensional coordinate and altitude. A prediction is performed by the algorithm that enables the driver to slow down at a specified distance before the speed limit signs and curves. In a study by Graser et al. (2015), digital elevation models (DEMs), as a source of information for elevation data, is used to estimate the energy consumption in an EV. That paper demonstrates that the standard of elevation data is crucial in energy estimation. In a study by Jo et al. (2013), energy management system of an $\mathrm{EV}$ is optimised by considering the road geometry information as the most important factor. In order to improve accuracy, GPS information and on-board vehicle sensors such as accelerometer, wheel speed sensor and gyro were unified by a fixed-interval optimal smoother.

In this study, an energy consumption estimation model is developed to be used for management of EV fleets. An energy map-based FMS algorithm has not been developed before in the literature. The existing FMS algorithms work based on 'distance' between every two points, let say A to B. However, in this paper the idea of using the required 'energy' between A and B is investigated instead. The proposed energy map-based FMS not only considers the 
road distance but also other factors such as road grade, vehicle dynamics, and driver model to have a better estimation of the required energy when going from A to B. Such an energy modelbased FMS has not existed yet in the market and we believe the results of this study contribute to the development and application of such FMS for future generation of autonomous EV fleets.

The second main contribution of this study is the analysis of energy estimation's accuracy in a larger scale, i.e. a fleet of vehicles. This is very important to know how much the performance of a FMS is sensitive to energy consumption estimation accuracy. For this investigation, the proposed FMS algorithm is simulated repeatedly by considering different levels of uncertainty. The uncertainty is quantified in terms of the tolerance in energy estimation error in $\mathrm{kWh}$. This study contains interesting results showing the influence of the level of energy estimation uncertainty on fleet's performance.

The estimation model is proposed for a typical EV in the market, i.e. Nissan LEAF. Real field tests have been conducted to evaluate performance of the proposed model. For this purpose, specific routes are considered and terrain specifications are extracted in the model as well. The simulation models are developed using MATLAB/Simulink based on real data of the vehicle, terrain and driver. The simulation results are then validated against the experimental field tests. Finally, a FMS algorithm is developed and simulated. In a case study, a fleet of EVs is simulated by considering the required energy which is needed to travel between points in a surrounded area.

\section{Mathematical Modelling for Energy Estimation}

Mathematical modelling for vehicle energy consumption estimation is a common technique in the literature that was originally developed for conventional vehicles and was used in a number of previous studies (Hayes et al. 2011, Delgado et al. 2012, Guzzella \& Sciarretta 2013). In this section, a mathematical model is constructed to simulate movement of a typical $\mathrm{EV}$ on a specific route. The main goal is to estimate the energy which is consumed for each journey before performing the journey itself. Such an energy consumption estimation model can be used in energy management systems of individual EV users or it might be used in larger scale for management of a fleet of EVs. This study is particularly focused on application of such an energy estimator in a FMS. The proposed mathematical model contains three main parts: 1) Terrain model, 2) Driver model, and 3) EV model. Combination of a map-based terrain model with the EV model is something new that is performed in this study specifically for energy consumption estimation in a FMS. 


\subsection{Terrain model}

Terrain model means all the route specifications that affect vehicle energy consumption. This mainly includes fixed road features such as junctions, traffic lights, speed limits, road gradient (slope) and variable features such as traffic condition, weather condition, road surface condition, etc. In this study, only some of these effective factors are considered and the rest are left for future works. Two main components of the terrain model in this study are elevation and speed profiles over the route (the first block on top left in Figure 1). Both profiles were gathered using a GPS phone application which records and stores longitude, latitude, altitude, distance and velocity of the vehicle at the sampling rate of $1 \mathrm{~Hz}$. The velocity profile reflects traffic condition and speed limits. In fact, a velocity profile is the result of many factors coming from the terrain specifications and also from the driving style (the driver model is discussed in the next part). The speed profile is directly obtained from the GPS app however, the road gradient should be calculated based on point-by-point GPS altitude data. To find the elevation between each two points, the difference between elevation (altitude) of the second point and the first point is divided by the change in horizontal distance between the two points. A positive gradient indicates uphill and a negative one represents a downhill.

The other components of the terrain model are road surface condition, weather condition (rainy and/or windy), speed limits, traffic lights, junctions, distance, traffic condition, etc. Although, all of these factors are not investigated separately in this study, their effect on EV energy consumption is considered to some extent. For example, the rolling resistance coefficient in the model represents the road surface. Weather condition is recorded in all tests however, the effects of wind or rain are not modelled (this is discussed more in Section 3.4). The speed limits, traffic lights, junctions and traffic condition are considered when the speed profile is recorded. It is believed that all these effects are included in the speed profile as a result of terrain specification. Finally, the travelled distance is also recorded by the GPS app and is considered in the model.

\subsection{Driver model}

In order to model a driver, a speed-tracking PID controller is used based on previous studies in literature (Song and Luo, 2014, Chen et al. 2013, Hayes et al. 2011). The driver model is aimed to follow a reference velocity profile that is driven by a human driver during the tests. In the simulation environment, the difference between the vehicle velocity and the reference velocity is called the tracking error, which is the input to the PID controller. The output of the PID controller is a command in response to the error value which can be either an acceleration 
or a brake command. The output command is normalized between 0 and 1 that means 'released' or 'fully-pressed' pedal status, respectively. In order to tune the PID controller gains, ZieglerNichols technique is used; this involves increasing gains until the onset of instability is reached, then using the information derived from this to select parameters (Ziegler et al. 1942). The driver model is shown as a single block in Figure 1. The performance of the proposed controller and simulation results are discussed later in Section 3.4.

Referring to the literature, driving style varies from person to person. This is important since it can affect EV's energy consumption. Since, this topic has been investigated deeply in a number of previous studies in literature such as Vatanparvar et al. (2018) and Corti et al. (2013), it is not repeated here again. In this study, in order to minimise the effect of driving style variations on EV energy consumption, the same driver has performed all the experiments. In this way, a 'consistent' driving style was assured. The goal here is to prove the proposed energy estimation concept and the results are obtained for one driver however, the same approach and procedure can be repeated for any number of drivers. Regarding the implication aspects, at the time being, fleet operators can continuously monitor their drivers and consequently, it would be clear which driver is lower/higher than average in terms of energy consumption when travelling the same route for the same time. That sort of data can be used to extract a 'modification factor' for each driver to generate a more realistic energy consumption value based on the average expected value (to be generated by the estimator).

\subsection{EV model}

This section focuses on the development of an EV model and its simulation under particular driving conditions (coming from the terrain and driver models). For this purpose, Nissan LEAF EV was selected as a popular vehicle with the most available data in literature. The model 2015 was selected as it was available for experimental tests. MATLAB/Simulink environment is used to realize the EV model as shown in Figure 1. The numerical data of the Nissan LEAF 2015 are listed in Table 1. Nissan LEAF 2015 has an $80 \mathrm{~kW}$ electric motor with maximum torque of 289 N.m. The required torque $\left(T_{r e q}\right)$ is determined with respect to the driver acceleration command $(A c c)$ :

$$
T_{\text {req }}=\text { Acc } \cdot T_{\max }
$$

where $T_{\operatorname{mmax}}$ is the maximum torque of the electric motor at a certain speed. In the drivetrain model, the required power is obtained from the required torque. The requested power $\left(P_{r e q}\right)$ is then sent to the battery model to see how much power is available: 


$$
P_{r e q}=T_{r e q} \omega^{*}
$$

where $\omega^{*}$ is the rotational velocity of the motor that is obtained by considering the vehicle speed and gear ratio

$$
\omega^{*}=\frac{\text { vehicle speed }}{R_{w}} G
$$

Available battery power is derived by considering the maximum current and voltage drop before hitting the cut-off voltage. Eventually, the tractive force $\left(F_{\mathrm{t}}\right)$ is updated based on the available torque $\left(T_{a v l}\right)$.

$$
F_{t}=\eta_{m} \cdot \eta_{g e a r} \cdot \frac{G \cdot T_{a v l}}{R_{w}}
$$

where $\eta_{m}$ is the efficiency of the electric motor (that is assumed to be constant), $\eta_{\text {gear }}$ is the efficiency of the gearbox, $R_{w}$ is the radius of the wheel, while gear ratio is shown as $G$.

The battery pack model consists of smaller components called cell. The required power is scaled down to calculate power demand from a single cell, assuming identical cells in the pack. After calculating the available power in a single cell, the power is again scaled up. Specifications of Nissan LEAF battery pack are listed in Table 2.

The vehicle speed is calculated by integrating the acceleration that is obtained as a result of the superposition of all forces applied to the vehicle including the traction force and all the opposing forces due to road gradient $\left(F_{g}\right)$, rolling resistance $\left(F_{\text {roll }}\right)$, aerodynamic drag $\left(F_{\text {aero }}\right)$ and brake. The total braking force is determined according to the driver's deceleration command. Brake system includes two parts: friction brake force $\left(F_{b f}\right)$ and regenerative brake force $\left(F_{b . r e g}\right)$. In the simulation model, the ratio of the total braking force $X$ is provided by the regenerative braking system while $(1-X)$ ratio is provided by the friction braking system. The parameter $X$ is not constant but it changes according to the velocity in a way to use more regenerative braking at high speeds (when more kinetic energy is available). 


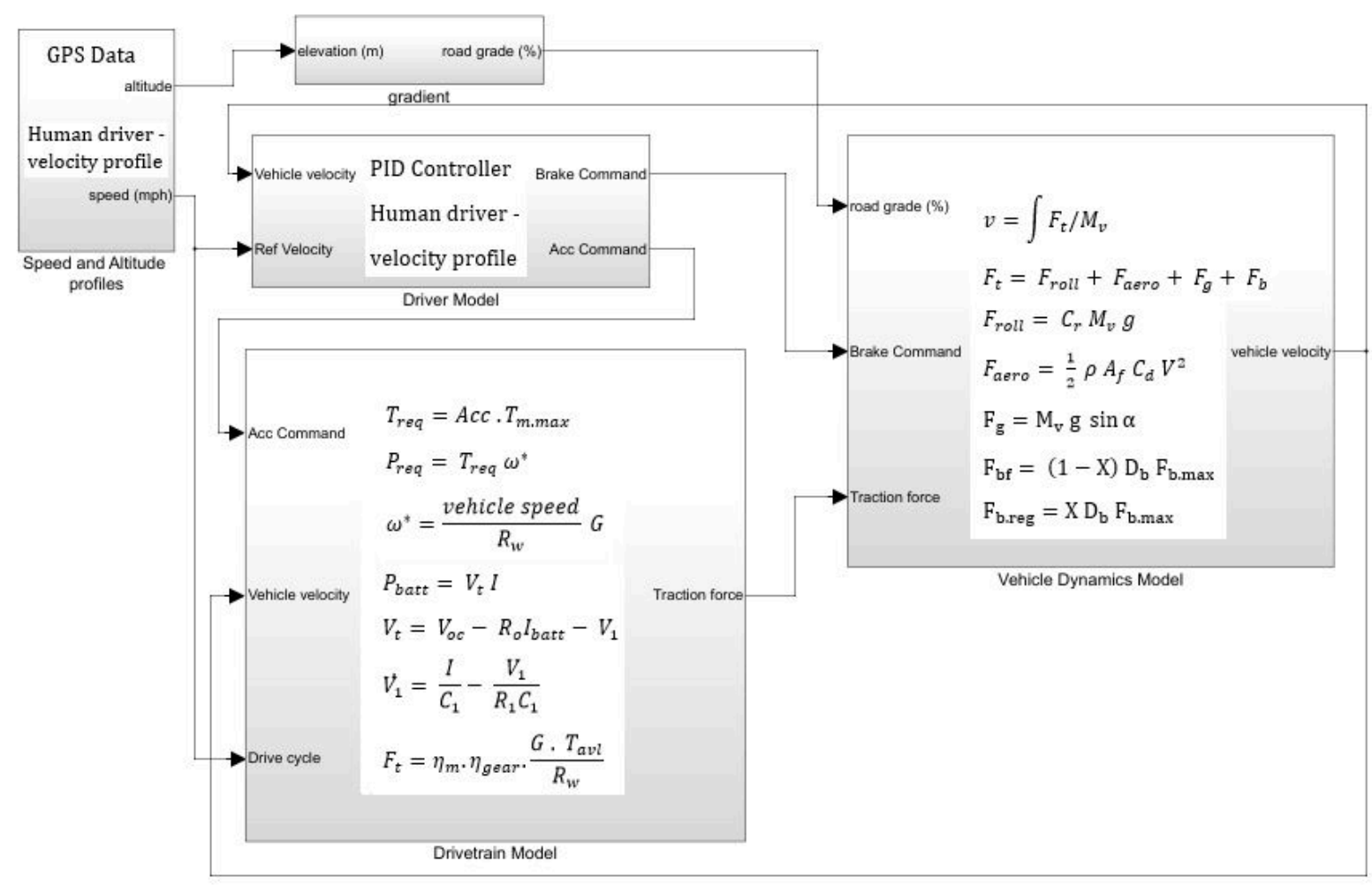

Figure 1: Simulink model developed for energy estimation

Table 1: Nissan LEAF 2015 model parameters

\begin{tabular}{cccc}
\hline Parameter & Description & Value & Unit \\
\hline $\boldsymbol{A}_{\boldsymbol{f}}$ & Vehicle frontal area & 2.27 & $\mathrm{~m} 2$ \\
$\boldsymbol{C}_{\boldsymbol{d}}$ & Drag coefficient & 0.29 & - \\
\hline $\boldsymbol{C}_{\boldsymbol{r}}$ & Rolling resistant coefficient & 0.008 & - \\
\hline $\mathbf{g}$ & Gravity & 9.81 & $\mathrm{~m} / \mathrm{s} 2$ \\
\hline $\boldsymbol{G}$ & Gear ratio & 7.94 & - \\
\hline $\boldsymbol{M}_{\boldsymbol{v}}$ & Vehicle's mass & 1500 & $\mathrm{Kg}$ \\
\hline $\boldsymbol{R}_{\boldsymbol{w}}$ & Wheel's radius & 0.316 & $\mathrm{~m}$ \\
\hline $\boldsymbol{\rho}$ & Air density & 1.225 & $\mathrm{~kg} / \mathrm{m} 3$ \\
\hline $\boldsymbol{\eta}_{\boldsymbol{m}}$ & Inverter and electric & 0.88 & - \\
\hline $\boldsymbol{\eta}_{\boldsymbol{g e a r}}$ & motor's efficiency & & - \\
\hline $\boldsymbol{F}_{\boldsymbol{b . m a x}}$ & Gearbox efficiency & 0.97 & $\mathrm{~N}$ \\
\hline $\boldsymbol{\eta}_{\boldsymbol{r e g e n}}$ & Maximum brake force & 3500 & - \\
\hline
\end{tabular}


Table 2: Nissan LEAF battery parameters

\begin{tabular}{cc}
\hline Parameter & Value \\
\hline Number of cells in series & 98 \\
Number of parallel circuits & 2 \\
Voltage of cell (V) & 4.2 \\
Capacity of cell (Ah) & 32.5 \\
Capacity of battery pack (KWh) & 24 \\
Cell cut off voltage (V) & 2.5 \\
\hline
\end{tabular}

\section{Experimental Tests and Simulation Model Verification}

Experimental tests are conducted to validate the proposed energy estimation model constructed in Section 2. For this purpose, two Nissan LEAF EVs are tested on four routes. The test routes are selected to cover motorway, urban, rural and intercity areas. The vehicle movement and its energy consumption are recorded at a fixed sampling rate of $1 \mathrm{~Hz}$.

\subsection{Test equipment}

The test equipment which is used in this study mainly contains the following items:

- EV, i.e. Nissan LEAF 2015

- GPS phone application to record EV position and velocity

- Dash camera, 12-volt charger and a memory card to temporary record of EV dashboard information (mainly for recording battery SoC)

- PC/laptop for data storage and analysis

Besides being very popular and having most data available in literature, Nissan LEAF has this option to see the battery SoC value on the dashboard with the accuracy of $1 \%$ which makes this car suitable for our investigations. Two rented cars are used in experiments for data gathering. Because of the restrictions of rental cars such as limited access time and prohibition to change anything in the vehicle, test equipment is selected to be easily installed and able to record the required data on the vehicle's dashboard. Figure 2 shows one of the Nissan LEAF vehicles that were used for the experiments in this study, and the information displayed on the vehicle's dashboard. The GPS phone application is utilized to record the vehicle position, road elevation, travelled distance and velocity, using the sampling rate of $1 \mathrm{~Hz}$ in format of a .gpx file. A camera, GZDL Full HD 1080P Mini Dash Cam Car (shown in Figure 2), is used to record the vehicle's dashboard information with the focus on battery SoC. The ScanDisk Ultra 
32GB micro SDHC memory card inside the camera was used to save the data for around seven hours continuously.
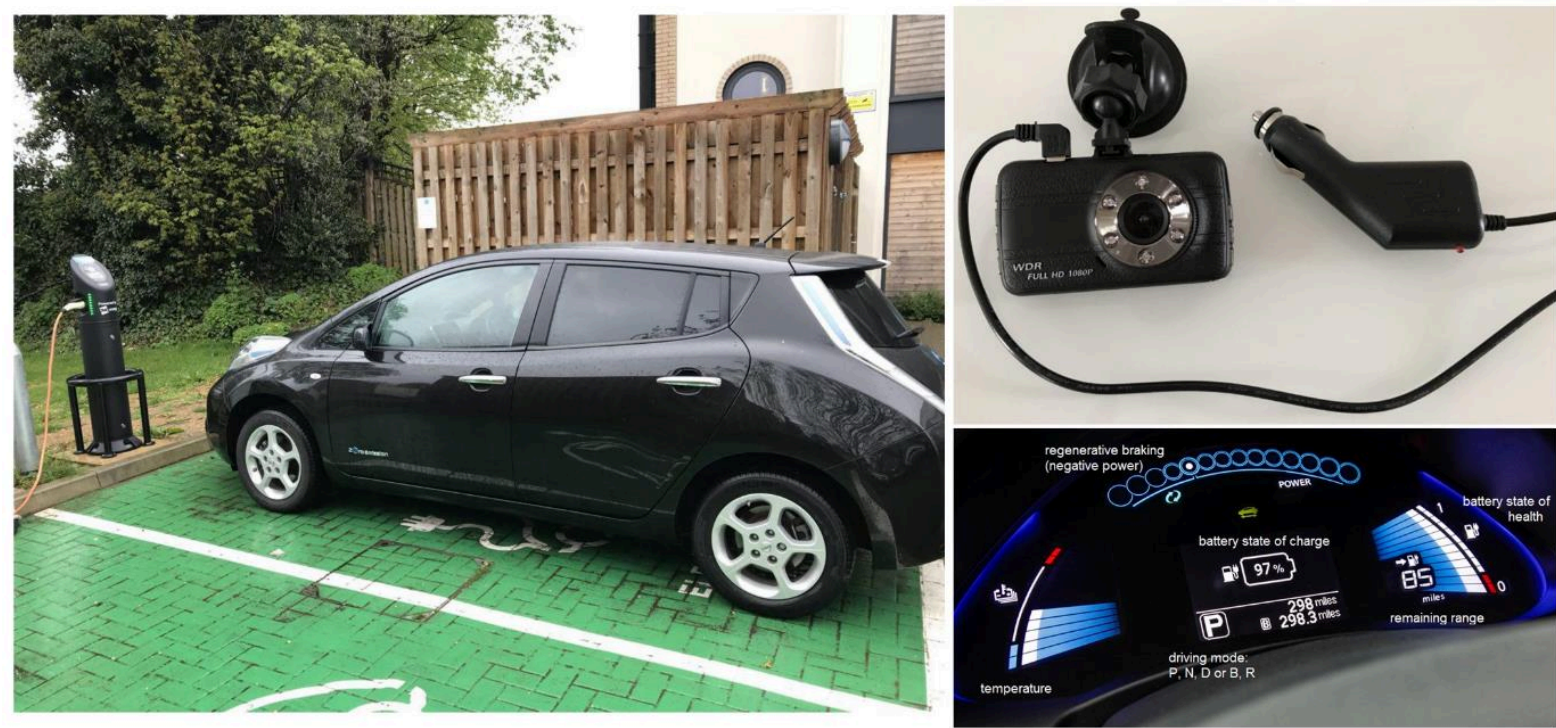

Figure 2: Nissan LEAF 2015 that was used in experimental tests

\subsection{Driving route selection}

Four routes were considered for data acquisition including a variety of different sections of motorway, urban and rural roads. Another factor for route selection was the location of the depot of the rented EVs which was in Dunstable, a town in the UK. As a result, the following routes were selected by considering the range of $\mathrm{EV}$ :

- Dunstable to Milton Keynes: The length of this route is $31.2 \mathrm{~km}$, and it mainly contains high speed driving in motorway (M1) as shown in Figure 3-(a).

- Milton Keynes to Cranfield: The length of this route is $8.26 \mathrm{~km}$, and it mainly contains urban and rural areas as show in Figure 3-(b).

- Cranfield to Milton Keynes: This route has exactly the same features as the second route, however the road elevation is the opposite (uphill and downhill).

- Milton Keynes to Dunstable: The length of this route is $26.3 \mathrm{~km}$. To include different types of roads, a completely different route was selected to return to Dunstable from Milton Keynes as shown in Figure 3-(c). This route includes motorway and urban driving. 

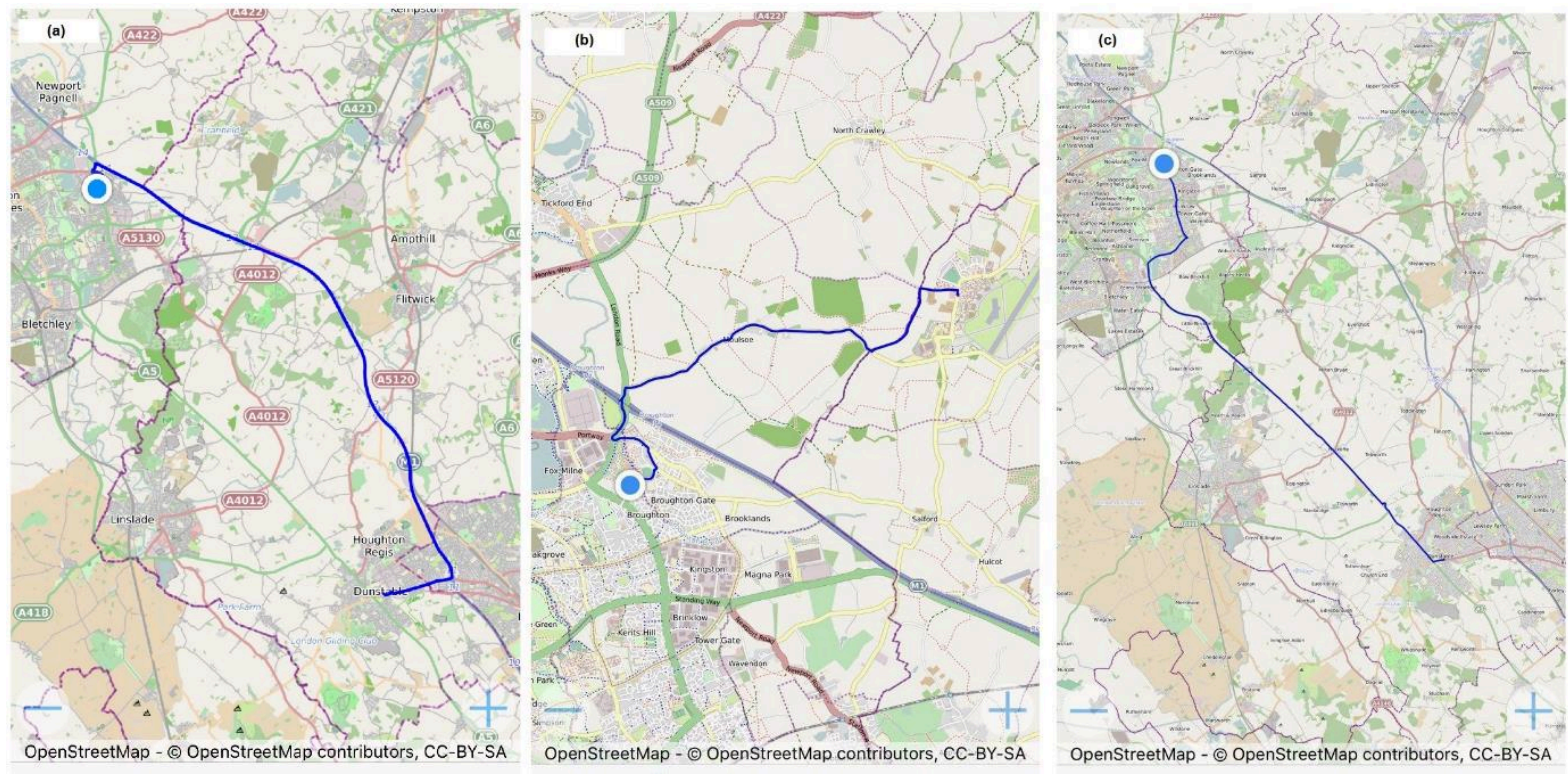

Figure 3: Test routes: (a) from Dunstable to Milton Keynes, (b) from Milton Keynes to Cranfield and return, (c) from Milton Keynes to Dunstable

\subsection{Test data}

The data recorded in our tests is determined by considering the final goal of this study which is the energy consumption estimation. So, every parameter that is effective on the vehicle energy consumption, should be considered and recorded. Some of these parameters are recorded at the beginning of each test manually. However, some other parameters need to be recorded during the test using the equipment.

In this study, the following parameters were observed and recorded before each test:

- Vehicle (Nissan LEAF 2015 EVs): The exact model of the car was checked and the corresponding data from the car manual such as vehicle's weight, battery capacity, etc. were extracted. Vehicle's weight of this model is around $1500 \mathrm{~kg}$. Battery capacity of this model is $24 \mathrm{kWh}$ that is used for energy consumption calculation.

- Passenger and baggage: exact weight of the passengers and their baggage were recorded in each test that was around $140 \mathrm{~kg}$ in total.

- Battery state-of-health (SoH): Information of the SoH is important since the battery capacity is used for the energy consumption calculation. In all the tests, battery SoH was assumed to be $100 \%$ (fresh battery).

- Eco Mode: Nissan LEAF has an Eco Mode that can be set ON or OFF. When it is ON, the acceleration will be limited to save energy. In all the tests, the Eco Mode was OFF to allow a normal driving performance. 
- Regenerative braking/driving mode: Nissan LEAF has two different modes of driving, D and B modes. While both modes have regenerative braking, it is applied more in B mode. In all the tests, D mode was used.

- Temperature control system: air-conditioning system or temperature control system was OFF during all the tests performed in this study. The reason for that was to avoid the complexities coming from energy consumption by this system. There are many studies in literature that had particularly investigated the energy consumption of a vehicle air-conditioning system.

- Tyre pressure: since tyre pressure can affect vehicle energy consumption, this was checked before each test, to make sure that it is within the standard normal range.

In addition to the above data, GPS App and the camera record other parameters such as date, time, vehicle's position, road elevation, travelled distance, vehicle's speed and the vehicle dashboard information like battery SoC. The battery SoC and other useful information are manually extracted from the camera videos after the test. It is important to synchronise the camera data and the GPS data for accurate analysis of energy consumption. To do this, the absolute time from the clock which is available in both camera and GPS is used.

\subsection{Data analysis and model validation}

In this section all tests results are presented and analysed. They are also compared with simulation results obtained from the model developed in Section 2.

As mentioned in Subsection 3.2, four routes are considered in road testing. The EVs are rented in Dunstable so, the first test route is from Dunstable to Milton Keynes. Then there was a journey from Milton Keynes to Cranfield and return, and finally a trip from Milton Keynes back to Dunstable. Each complete test contains all the above mentioned routes in the same sequence. Four complete tests were carried out using two EVs (two tests for each vehicle) under the following conditions:

- All tests were performed by the same driver.

- All tests were performed on Sunday morning from 10.00 am to $12.00 \mathrm{pm}$.

- All tests were started with the battery at a fully charge state (initial battery SoC was between $97 \%$ and $100 \%$ ).

- In all tests, the EV Eco mode was OFF to have a normal driving. 
- In all tests, the vehicle air conditioning system (temperature control system) was OFF in order to minimize the effect of ancillary power use in the car.

Table 3 contains the main outcomes of each test including the duration of tests, travelled distance, initial and final battery SoC, and the changes in battery SoC that show how much energy is used in kWh. The energy consumption is calculated based on the battery's nominal capacity which is assumed to be constant at $24 \mathrm{kWh}$. So, for example, $50 \%$ change in SoC is equivalent to $12 \mathrm{kWh}$ energy consumption. In both EVs, the battery state-of-health $(\mathrm{SoH})$ indicator on the dashboard was showing $100 \%$, meaning that the original capacity (i.e. $24 \mathrm{kWh}$ ) is still available. We used that indicator as a reference battery SoH in our model. Every time we tested any of the EVs, that gauge was checked to confirm the battery health status. This is important because the battery nominal capacity is used in our energy consumption calculations. SoH of $100 \%$ means the battery is still fresh and the nominal battery capacity (published by the car manufacturer) can be used. In a case when SoH is less than $100 \%$, that percentage of the nominal capacity should be used instead.

As presented in Table 3, the whole journey starting from the charging point in Dunstable and returning to that point again, would consume different values of energy at different tests. The results are discussed in details in the followings. Actually, only two of those tests, i.e. EV 1 - Test 2 and EV 2 - Test 2, are considered as 'normal' in which around 52\% of the vehicle's battery SoC is consumed $(12.48 \mathrm{kWh})$.

In EV 1 - Test 1, while the test duration is normal, the energy consumption is more than other tests. The reason for this is because of the windy and rainy weather condition. Wind causes more drag resistant force that increases energy consumption. On the other hand, rainy weather condition caused the vehicle screen wiper to work continuously for two hours during the test. As a result, 19\% increase in energy consumption was observed in comparison to the expected 'normal' value (i.e. $12.48 \mathrm{kWh}$ ). Weather condition can also be considered as a modification factor in energy consumption estimation. Determination of the exact values due to rain or wind also is considered as future works.

In EV 2 - Test 1, the energy consumption is $13.2 \mathrm{kWh}$ that is a bit more than the expected 'normal' value. The reason is because the vehicle was facing more traffic on that day, as also reflected in the journey time. The time of the whole test is $5060 \mathrm{sec}$ for EV 2 - Test 1 that is longer than all other tests. The traffic was busier on Dunstable to Milton Keynes and return path rather than in Cranfield road. This increase in energy consumption is around 5.6\% more 
comparing to the expected value, i.e. $12.48 \mathrm{kWh}$. This result can be considered as a modification factor (e.g. 5\% added energy consumption due to traffic) in our estimations when traffic is predicted in that route. Nowadays, traffic data are available everywhere and technically it is not a challenging task to consider it for energy estimation before each journey in a fleet management system. Because of the limitations in doing more tests in this study, traffic condition was not investigated separately however, the results are showing reasonable increase in energy as expected.

Table 3: Nissan LEAF road test data

\begin{tabular}{|c|c|c|c|c|c|c|}
\hline Test & $\begin{array}{c}\text { duration } \\
\text { (sec) }\end{array}$ & $\begin{array}{c}\text { Travelled } \\
\text { distance } \\
\text { (km) }\end{array}$ & $\begin{array}{c}\text { Initial } \\
\text { SoC }\end{array}$ & $\begin{array}{c}\text { Final } \\
\text { SoC }\end{array}$ & $\begin{array}{c}\text { SoC } \\
\text { change }\end{array}$ & $\begin{array}{c}\text { Equivalent } \\
\text { energy } \\
\text { consumption } \\
\text { (kWh) }\end{array}$ \\
\hline EV1 - Test 1 & $\mathbf{4 8 7 3}$ & $\mathbf{7 4 . 0 1}$ & $\mathbf{9 8 \%}$ & $\mathbf{3 6 \%}$ & $\mathbf{6 2 \%}$ & $\mathbf{1 4 . 8 8}$ \\
\hline Dunstable to MK & 1653 & 31.12 & $98 \%$ & $76 \%$ & $22 \%$ & 5.28 \\
\hline MK to Cranfield & 741 & 8.35 & $76 \%$ & $68 \%$ & $8 \%$ & 1.92 \\
\hline Cranfield to MK & 743 & 8.14 & $68 \%$ & $61 \%$ & $7 \%$ & 1.68 \\
\hline MK to Dunstable & 1736 & 26.40 & $61 \%$ & $36 \%$ & $25 \%$ & 6.00 \\
\hline EV1 - Test 2 & $\mathbf{4 7 8 2}$ & $\mathbf{7 3 . 8 7}$ & $\mathbf{1 0 0 \%}$ & $\mathbf{4 8 \%}$ & $\mathbf{5 2 \%}$ & $\mathbf{1 2 . 4 8}$ \\
\hline Dunstable to MK & 1616 & 31.09 & $100 \%$ & $80 \%$ & $20 \%$ & 4.80 \\
\hline MK to Cranfield & 757 & 8.31 & $80 \%$ & $74 \%$ & $6 \%$ & 1.44 \\
\hline Cranfield to MK & 723 & 8.16 & $74 \%$ & $71 \%$ & $3 \%$ & 0.72 \\
\hline MK to Dunstable & 1686 & 26.31 & $71 \%$ & $48 \%$ & $23 \%$ & 5.52 \\
\hline EV2 - Test 1 & $\mathbf{5 0 6 0}$ & $\mathbf{7 3 . 7 3}$ & $\mathbf{9 9 \%}$ & $\mathbf{4 4 \%}$ & $\mathbf{5 5 \%}$ & $\mathbf{1 3 . 2 0}$ \\
\hline Dunstable to MK & 1757 & 31.11 & $99 \%$ & $79 \%$ & $20 \%$ & 4.80 \\
\hline MK to Cranfield & 726 & 8.24 & $79 \%$ & $72 \%$ & $7 \%$ & 1.68 \\
\hline Cranfield to MK & 714 & 8.07 & $72 \%$ & $65 \%$ & $7 \%$ & 1.68 \\
\hline MK to Dunstable & 1863 & 26.31 & $65 \%$ & $44 \%$ & $21 \%$ & 5.04 \\
\hline EV2 - Test 2 & $\mathbf{4 7 7 4}$ & $\mathbf{7 3 . 1 2}$ & $\mathbf{9 7 \%}$ & $\mathbf{4 5 \%}$ & $\mathbf{5 2 \%}$ & $\mathbf{1 2 . 4 8}$ \\
\hline Dunstable to MK & 1760 & 31.11 & $97 \%$ & $79 \%$ & $18 \%$ & 4.32 \\
\hline MK to Cranfield & 662 & 7.92 & $79 \%$ & $71 \%$ & $8 \%$ & 1.92 \\
\hline Cranfield to MK & 739 & 8.14 & $71 \%$ & $65 \%$ & $6 \%$ & 1.44 \\
\hline MK to Dunstable & 1613 & 25.95 & $65 \%$ & $45 \%$ & $20 \%$ & 4.80 \\
\hline
\end{tabular}

Figure 4 shows EV velocity vs. travelled distance during all complete tests (including all routes, starting and ending at Dunstable). Although the velocity profiles are a bit different due to traffic condition variation, they are very similar regarding the maximum/minimum limits, 
accelerations, etc. These similarities come from the road specifications (speed limits, etc.) and driving style which both were almost constant.

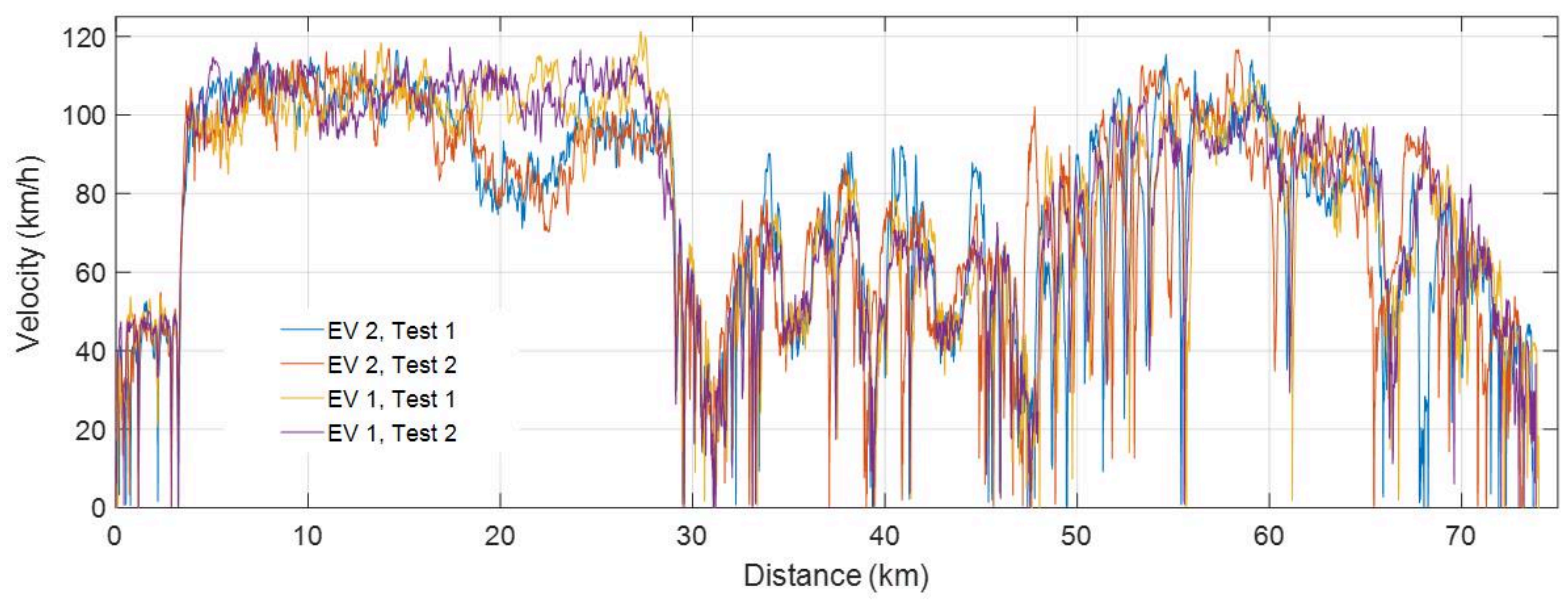

Figure 4: EV velocity vs. travelled distance during all complete tests

Figure 5 demonstrates the EV battery SoC variation vs. travelled distance during all complete tests (including all routes, starting and ending at Dunstable). The difference between SoC variations is caused by different factors. First of all, the initial SoC values were not exactly the same, changing within the range of $97 \%$ - 100\%. Secondly, energy consumption was affected by traffic condition and weather condition in two tests as discussed before.

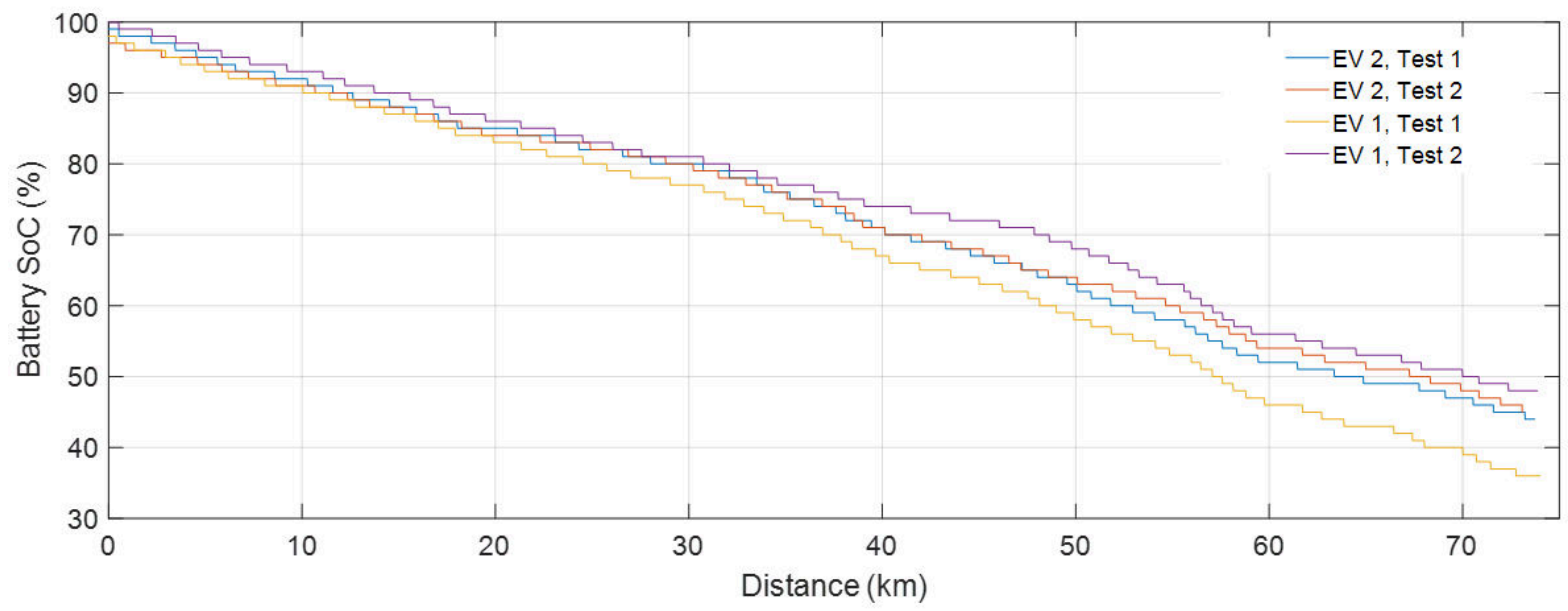

Figure 5: EV battery SoC vs. travelled distance during all complete tests

Table 4 demonstrates some comparisons between the test and simulation results. The road test data are used for model validation here. The error is defined as the difference between the real measured energy consumption during the tests and the energy consumption estimation obtained using the proposed simulation model. A negative error value means that the energy is underestimated. As listed in the table, the error value is less than $2.25 \%$ in three cases however, it reaches $12.65 \%$ in EV 1 - Test 1 . As mentioned above, the energy consumption during this 
test is more than other tests due to the windy and rainy weather condition. These two factors are not considered in the simulation, thus causing the higher estimation error. Except for that test, the model is working quite well in the other tests, i.e. prediction error less than $2.25 \%$.

Table 4: Nissan LEAF simulation results validation using test data

\begin{tabular}{|c|c|c|c|}
\hline Test & $\begin{array}{c}\text { Energy consumption } \\
\text { in test } \\
(\mathbf{k W h})\end{array}$ & $\begin{array}{c}\text { Energy consumption } \\
\text { in simulation } \\
\mathbf{( k W h )}\end{array}$ & $\begin{array}{c}\text { Estimation } \\
\text { error } \\
(\boldsymbol{\%})\end{array}$ \\
\hline EV1 - Test 1 & 14.88 & 13.00 & -12.65 \\
\hline EV1 - Test 2 & 12.48 & 12.76 & 2.25 \\
\hline EV2 - Test 1 & 13.20 & 13.15 & -0.41 \\
\hline EV2 - Test 2 & 12.48 & 12.50 & 0.20 \\
\hline
\end{tabular}

In order to present the simulation results, EV1-Test 2 is selected as the test case. All the following results are presented for that particular test however, similar trends exist in other tests. For example, Figure 6 demonstrates EV battery SoC during EV1-Test 2, obtained from two different sources: 1) simulation model and 2) EV dashboard. As shown in the figure, there is a good match between the simulation and test results that validates the model.

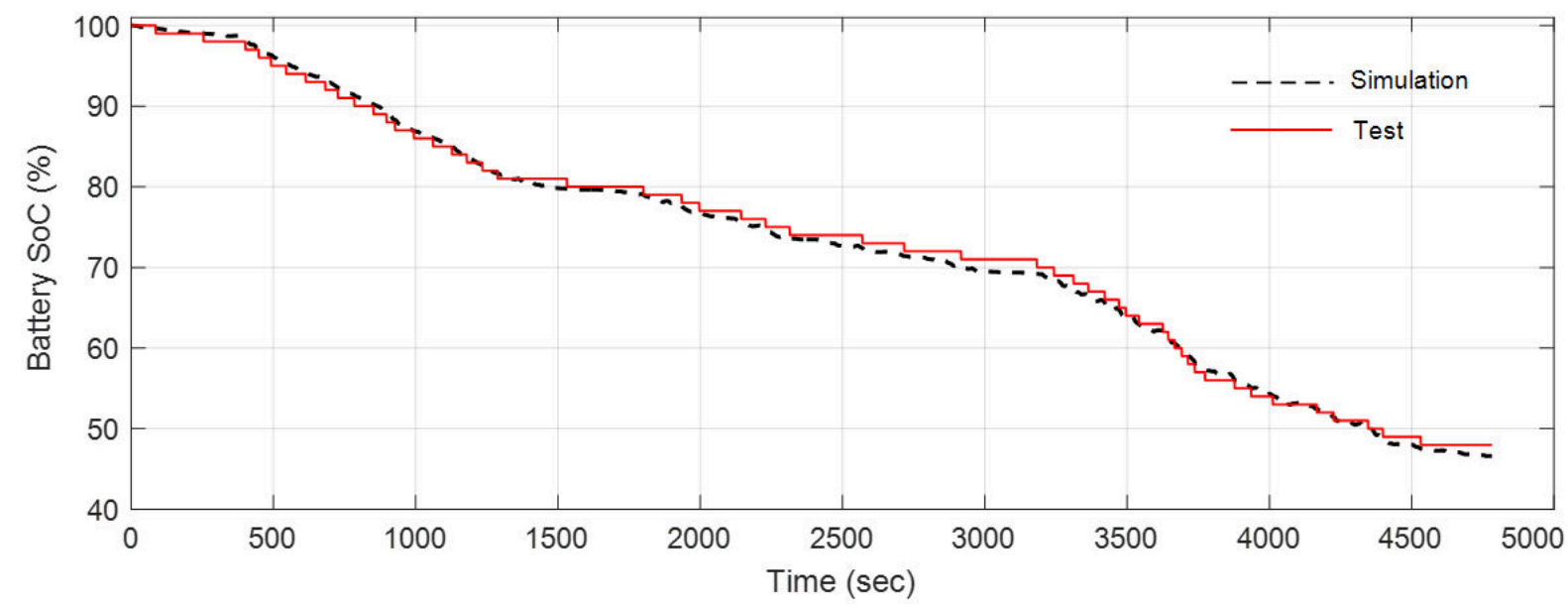

Figure 6: EV battery SoC during EV1-Test 2, obtained from simulation model and EV dashboard

Figure 7 depicts EV velocity during EV1-Test 2, obtained from two different sources: 1) simulation model (using the proposed PID controller as the driver model) and 2) GPS data recorded during the test. The test contains all four routes, starting from Dunstable, going to Milton Keynes, then to Cranfield and return. There are two velocity profiles for each route: 1) one is the recorded velocity (using GPS) that is imported to the simulation environment as the reference velocity, and 2) EV velocity that is obtained from the simulation model using the driver model that follows the reference speed. 
Figure 8 is showing EV battery current and terminal voltage, respectively, during EV1-Test 2. Both plots are obtained from the simulation model. Because these parameters were not directly measured during the tests, it is not possible to validate them directly. However, integration of the current (Coulomb counting) that gives the battery SoC, matches quite well the experimental data as shown in Figure 6. The maximum current is $180 \mathrm{~A}$ that is correct since each parallel circuit in the pack can deliver up to 90A and there are two parallel circuits in it. Voltage of the battery pack also looks reasonable referring back to the number of cells in series (i.e. 96 cells). The voltage drop due to the discharge current, depends on the battery internal resistance that is the least at very high $\mathrm{SoC}$ and then it increases gradually. Referring back to Table 3, we know that the battery SoC has changed from $100 \%$ to $48 \%$ during that test.

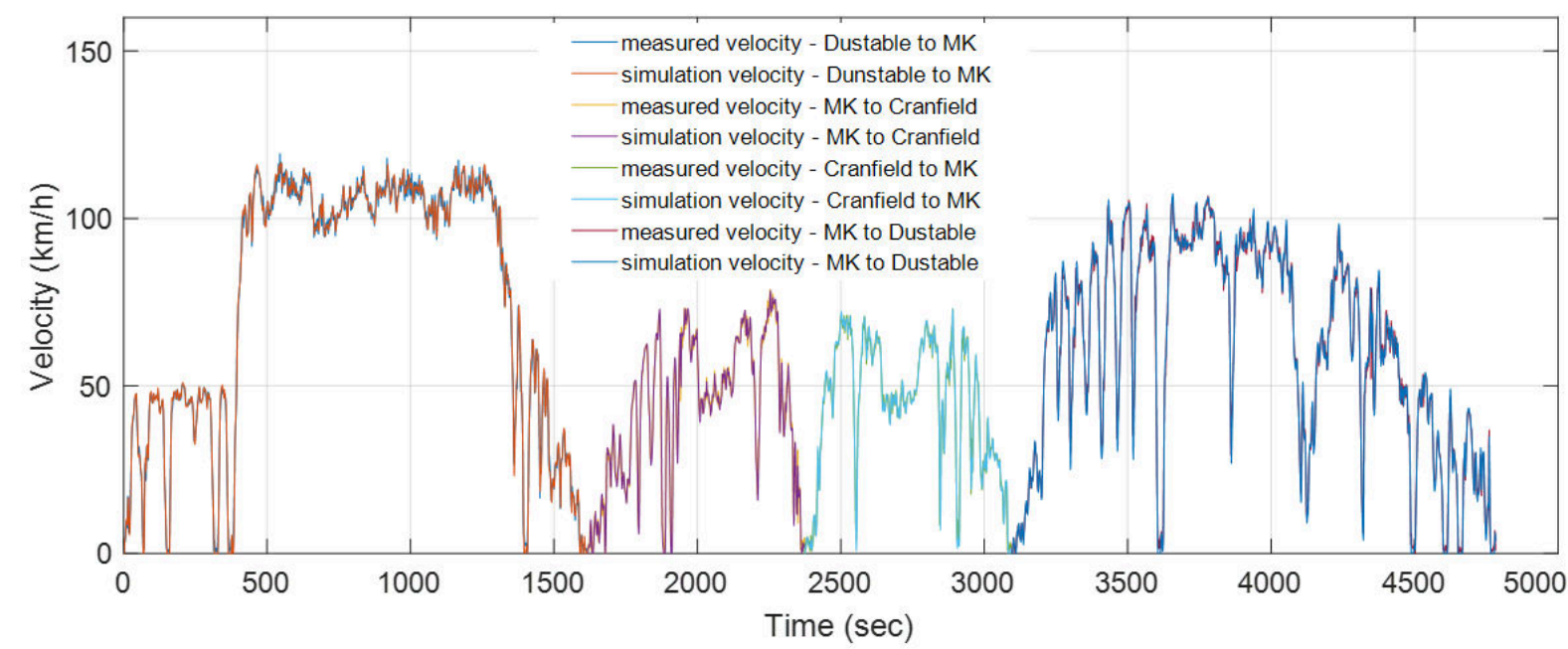

Figure 7: EV velocity during EV1-Test 2, obtained from simulation (using PID controller as the driver model) and GPS data 
(a)

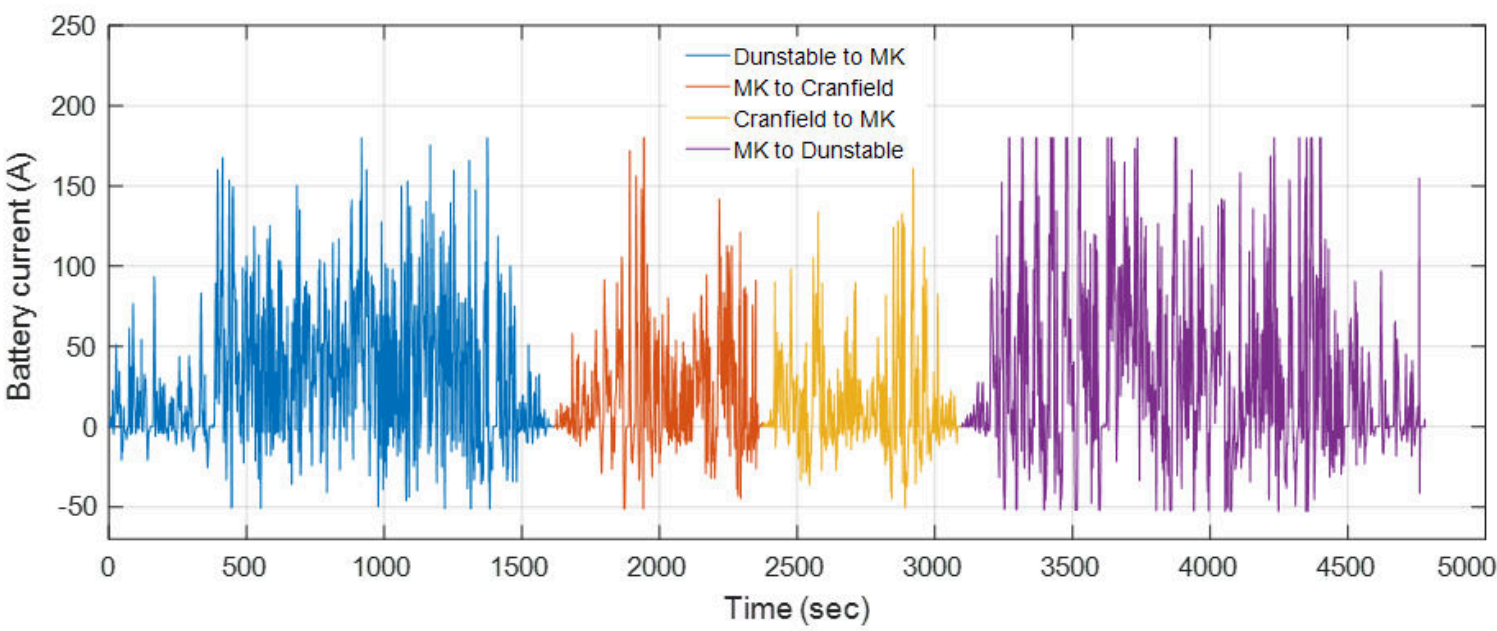

(b)

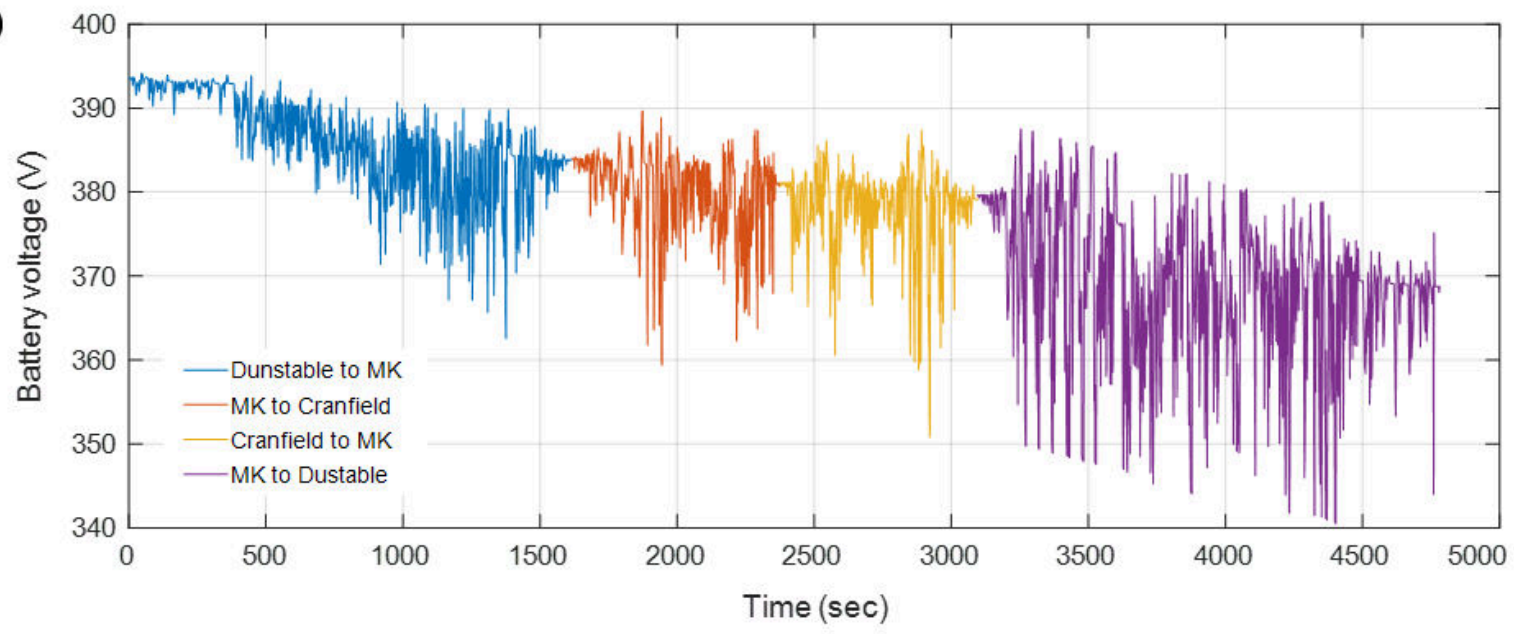

Figure 8: EV battery current and voltage during EV1-Test 2, obtained from simulation model

\section{Fleet Management System for EVs}

This study focuses particularly on development of a FMS to be used for a fleet of commercial EVs to do delivery tasks in a certain area. Such a FMS problem had been addressed in literature before (see for instance Fotouhi et al. 2016) but without taking into account the concept of energy. In the proposed FMS in this study, the 'energy' required to travel from let say point A to point B is considered instead of the 'distance' between them. Actually, the concept of energy not only contains the effect of distance but also it includes other features of the route, vehicle and driver. The results from the previous sections where an EV energy consumption estimator was developed, is applied in this section. In fact, the energy consumption estimator contributes to the accuracy of EV range estimation by considering the features of route, vehicle and driver in a FMS. In order to verify performance of the proposed algorithm, a simulation case study is considered as explained next. 


\subsection{Case study}

In this case study, a delivery vehicle routing problem is considered where a number of target locations are set to be reached by a fleet of EVs. The EVs should be managed in a way that they have enough charge to return to a charging depot after getting as many target points as possible. So the proposed FMS algorithm schedules and coordinates the trips of all EVs to cover all the target locations. The assumptions are: (i) the target locations are fixed and are available a day before, so this is not a real-time scheduling like taxi booking, and (ii) all EVs would return to the charging depot after finishing their tasks.

In this case study, one hundred target points are distributed randomly in a squared area with dimension of $(50 \times 50) \mathrm{km}^{2}$ as shown in Figure 9. The origin point $(\mathrm{x}=0, \mathrm{y}=0)$ of Cartesian coordinate is considered to be in the left bottom corner. The depot is assumed to be at the centre of the area $(x=25, y=25)$. The FMS problem is defined to get all the 100 points by using any number of EVs but trying to minimise the overall mileage of the fleet. To solve this problem, first we need to know the energy that is required to travel from each single point to another. The outcome of this analysis would be a $100 \times 100$ matrix in our case study (since we assumed to have 100 target points) including all energy values (in $\mathrm{kWh}$ ) from each point to all other points:

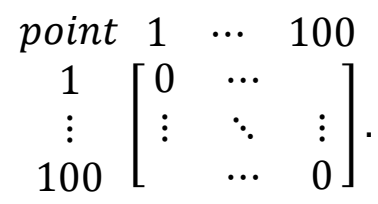

Such a matrix can be completed and regularly updated using the proposed energy consumption estimator. Because here the FMS is not going to be designed for a specific city or area, the locations of the points are randomly distributed. The energy values are calculated based on the distance between each two points and also the gradient between them. The gradient represents the altitude difference or elevation between the two points. For the sake of simplicity, a constant gradient of 0.005 is considered in the area shown in Figure 9. The elevation of each point ( $z$ value) is proportional to the distance of that point to the origin $(0,0)$. So, the lowest point $(z=0)$ is the origin and the highest elevation at $(50,50)$ is:

$$
z_{\text {max }}=0.005 \times \sqrt{(50-0)^{2}+(50-0)^{2}}=0.3536
$$

For any other point $\left(x_{i}, y_{i}\right)$, the elevation $\left(z_{i}\right)$ is obtained as follows:

$$
z_{i}=0.005 \times \sqrt{x^{2}+y^{2}}
$$


The gradient of 0.005 is a reasonable number since it also matches with the data obtained from the measurements presented before. The most elevation change happened in the route between Milton Keynes and Cranfield where the elevation changes around $40 \mathrm{~m}$ over $8 \mathrm{~km}$ distance as shown in Figure 10.

$$
\text { Average elevation }=\frac{40}{8000}=\frac{5}{1000}
$$

Having the distance and elevation between all 100 points, the energy consumption values between each pair of points is calculated as presented in Table 5.

The average energy consumption obtained in the field tests was $179 \mathrm{Wh}$ per $\mathrm{km}$. In order to get a better understanding about this number, we calculate the energy due to distance between the furthest pair of points (i.e. the origin and the point on right top corner):

$$
E_{d-\max }=0.179 \times \sqrt{50^{2}+50^{2}}=12.66 \mathrm{kWh}
$$

This energy is a bit more than a half of the Nissan LEAF battery pack's capacity (i.e. $24 \mathrm{kWh}$ ).

To consider the effect of gradient, a modification factor is added to the energy calculation based on a gradient factor $(G F)$ and the difference in elevations between the two points. So, we have:

$$
\begin{gathered}
E=E_{d}+E_{g} \\
E_{d}=0.179 \times \text { Distance } \\
E_{g}=G F \times(\text { elevation } 2-\text { elevation } 1)
\end{gathered}
$$

Choosing $G F=10$, the second term of energy $\left(E_{g}\right)$ would change between 0 and $3.536 \mathrm{kWh}$ (i.e. $G F \times z_{\max }$ ) depending on the distance between the two points. So the dominant term in the energy consumption is $E_{d}$, however the smaller term due to gradient $\left(E_{g}\right)$ would be also considered. 


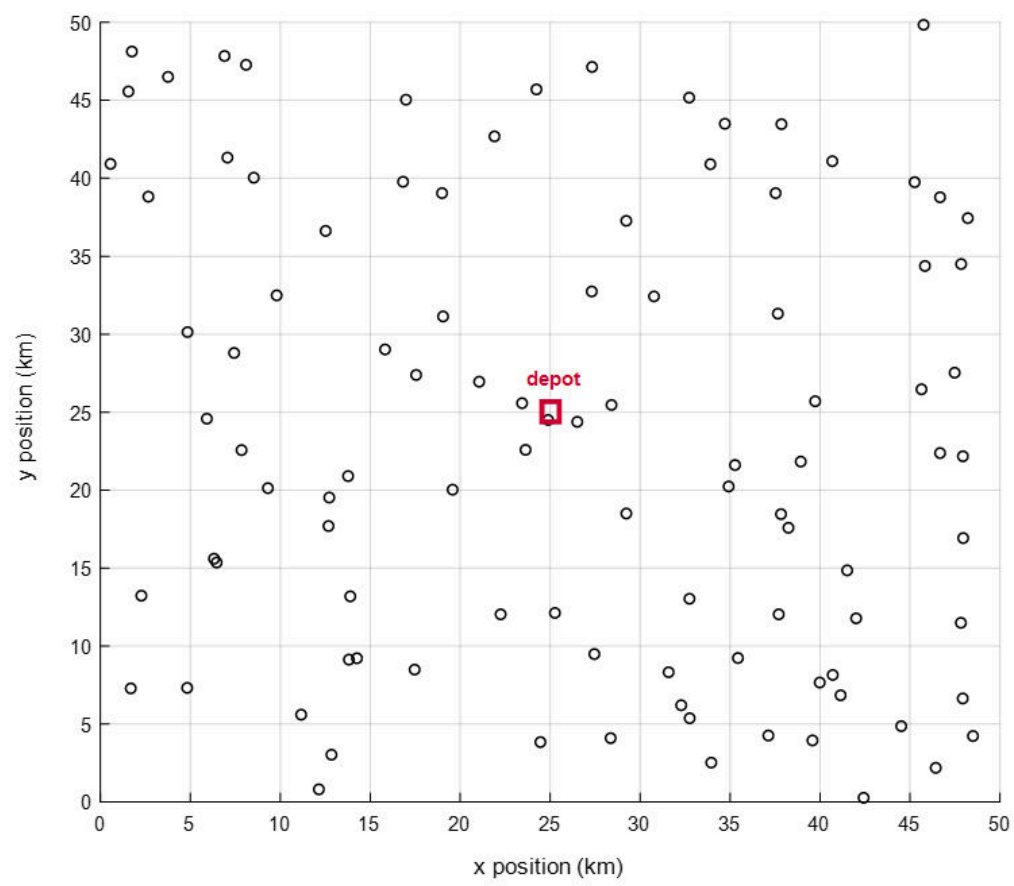

Figure 9: Random target locations and depot in the centre

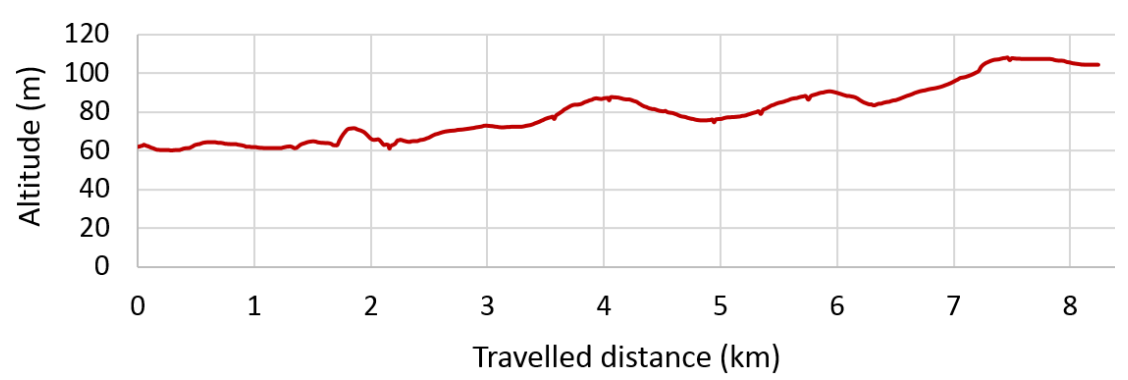

Figure 10: Variation of altitude vs. distance in route from MK to Cranfield

Table 5: Energy matrix for a case study including 100 target points

\begin{tabular}{|c|c|c|c|c|c|c|c|c|}
\hline $\begin{array}{c}\text { Point } \\
\text { No. }\end{array}$ & 1 & 2 & 3 & 4 & 5 & $\ldots$ & 99 & 100 \\
\hline 1 & 0.0 & 7.4 & 5.9 & 4.4 & 1.4 & $\ldots$ & 10.5 & 8.1 \\
\hline 2 & 5.5 & 0.0 & 7.1 & 2.3 & 5.6 & $\ldots$ & 8.1 & 4.9 \\
\hline 3 & 8.4 & 11.4 & 0.0 & 10.1 & 6.1 & $\ldots$ & 6.5 & 6.7 \\
\hline 4 & 3.3 & 3.1 & 6.5 & 0.0 & 3.6 & $\ldots$ & 9.0 & 5.9 \\
\hline 5 & 2.3 & 8.3 & 4.5 & 5.6 & 0.0 & $\ldots$ & 9.5 & 7.6 \\
\hline$\ldots$ & $\ldots$ & $\ldots$ & $\ldots$ & $\ldots$ & $\ldots$ & $\ldots$ & $\ldots$ & $\ldots$ \\
\hline 99 & 10.5 & 10.0 & 4.1 & 10.2 & 8.7 & $\ldots$ & 0.0 & 3.4 \\
\hline 100 & 7.9 & 6.6 & 4.0 & 6.9 & 6.5 & $\ldots$ & 3.2 & 0.0 \\
\hline
\end{tabular}




\subsection{Fleet management algorithm}

In this section, a FMS algorithm is developed to plan the trips of EVs to reach all target points. It is obvious that the number of EVs that are required to reach all the target points depends on the number of points, their locations and also the range of EVs in general. In the proposed FMS algorithm, there is no limitation for the maximum number of EVs; thus the vehicles are dispatched until all the tasks are completed. The proposed trip planning algorithm works based on the nearest neighbour algorithm. The first EV starts from the best initial point (which is determined in Step 2 in the algorithm), and then goes to the nearest point next to the first point, and so on. This process continues until the battery charge of the first EV is low, which means not enough to return to the depot. At this state, the first EV's plan is completed. It then returns to the depot and the remaining points will be covered by other vehicles. The second EV starts from the best initial point among the remaining target points and its journey continues until the battery is low charged. More EVs will be dispatched if it is required until all the target points are covered. The proposed algorithm can be summarized as follows:

Step 1: All EVs are fully charged at depot before starting the journeys,

Step 2: A vector of target points and the best initial point are allocated to each EV based on the EV range and demanded energy calculations,

Step 3: Route planning of EVs is performed according to the nearest-neighbour procedure explained by Fotouhi et al. (2016) until all the allocated target points are covered.

One drawback of this algorithm is its dependency on the starting point. A solution to manage this limitation is to try all points as initial condition (100 points in this case) and the algorithm runs many times to find the best point to start. The best initial point is determined based on an objective function $(f)$ :

$$
f\left(X_{\text {best init }}\right)=\operatorname{Min}\left(f\left(X_{i}\right)\right), \text { for } i=1 \cdots n
$$

where $X_{\text {best init }}$ is the best initial point, $X_{i}$ is a target point, $n$ is the number of all target points ( $n=100$ in this case). The function $f$ is the total energy consumed by all EVs using the proposed FMS algorithm:

$$
f\left(X_{i}\right)=\sum_{j=1}^{N_{\text {dispatch }}} E_{j}, \text { when starting from point } i
$$

where $E_{j}$ is the total energy consumed by the $j t h \mathrm{EV}$, and $N_{\text {dispatch }}$ is the number of EVs being dispatched. 
Since the algorithm is quite fast, running it many times does not create high computational cost. The time required to run the algorithm with 100 points is 0.28 seconds using a desktop PC with Intel(R) Core(TM) i7-4600U CPU 2.10GHz processor.

It should be noted that the proposed nearest neighbour FMS algorithm gives a quick, but not the optimal solution. There is no analytical method to obtain the 'optimal' solution of such a problem rather than the direct search method that tries all possible scenarios. However, direct search is not applicable when the number of points is quite high. Other optimisation techniques can also be applied, however none of them guarantees the global optima. Investigation of different optimisation techniques to be used in the FMS algorithm is out of the scope of this study. Regardless of what the FMS algorithm is (Nearest Neighbour, ACO, etc.), we need an accurate estimation of the required energy to travel between two points for instance from point A to point $B$. This paper focuses on this estimation problem rather than the optimisation algorithm itself. Another assumption in this study is considering only one charging depot at the centre that can be extended in future studies. When more charging stations are used, optimal locating of the stations would be another research area as discussed by (Gimenez et al. 2016). In addition, refuelling time (as discussed by Jeong \& Illades Boy, 2018) is not considered here since slow battery charging is used instead of fast charging or battery swapping.

\subsection{FMS simulation and analysis}

The proposed FMS is simulated for the case-study presented in Section 4.1. Figure 11 demonstrates the FMS simulation results for a case which is NOT started from the best initial point (just starting from a random point). In this case, seven EVs are dispatched to cover the 100 target points. As shown in the figure, all target points are finally reached by the FMS algorithm although this requires with more effort than needed. The total energy consumed by the fleet is $132 \mathrm{kWh}$ in this case. If the FMS starts from the best initial point, only five EVs are needed to cover all the target points and the total fleet energy consumption is only 112 kWh. The FMS simulation result using the best initial conditions is shown in Figure 12. Individual EVs' energy consumptions are listed in Table 6 which demonstrates that the proposed FMS algorithm works properly. The result shows that each EV's energy consumption is less than $24 \mathrm{kWh}$ which was considered as the battery capacity.

Referring back to the proposed EV energy consumption estimator, the importance of an accurate estimation is investigated at a larger scale here in a FMS. Such an estimator would use vehicle data, terrain data and driver data to generate an accurate EV energy consumption 
between each two points in FMS. Of course, the accuracy of any estimator is limited and some uncertainties are expected in this FMS calculation. The discussion here is to know how the uncertainties in the estimation would affect the overall FMS performance. For this investigation, the proposed FMS algorithm is run repeatedly by considering different levels of uncertainty. The uncertainty is quantified in terms of the tolerance in energy estimation in $\mathrm{kWh}$. For example, if the uncertainty in energy estimation is around $1 \mathrm{kWh}$, the effective range of the EVs in the fleet is considered to be $23 \mathrm{kWh}$ instead of $24 \mathrm{kWh}$. By this way, the FMS would overcome the uncertainty however, it is performing in a more conservative way. Similar simulation is done when the uncertainty is $2 \mathrm{kWh}$ by considering $22 \mathrm{kWh}$ energy for each $\mathrm{EV}$ in the fleet and so on. Table 7 contains interesting results showing the influence of the level of uncertainty (mainly due to lack of accuracy in energy estimation) on fleet's performance. The results demonstrate how significant the role of an energy estimator is in an EV FMS. In average, every one percent improvement in the estimation accuracy can improve the overall fleet energy consumption around one percent. This can be quite a significant amount of energy in a large fleet. In our case study of a small fleet with less than $10 \mathrm{EVs}, 1 \%$ improvement is equivalent to $1.1 \mathrm{kWh}$ for each operation. This can be scaled-up when the number of EVs is increased from 10 to 10,000 for example, that leads to $1100 \mathrm{kWh}$ per day (assuming one operation each day).

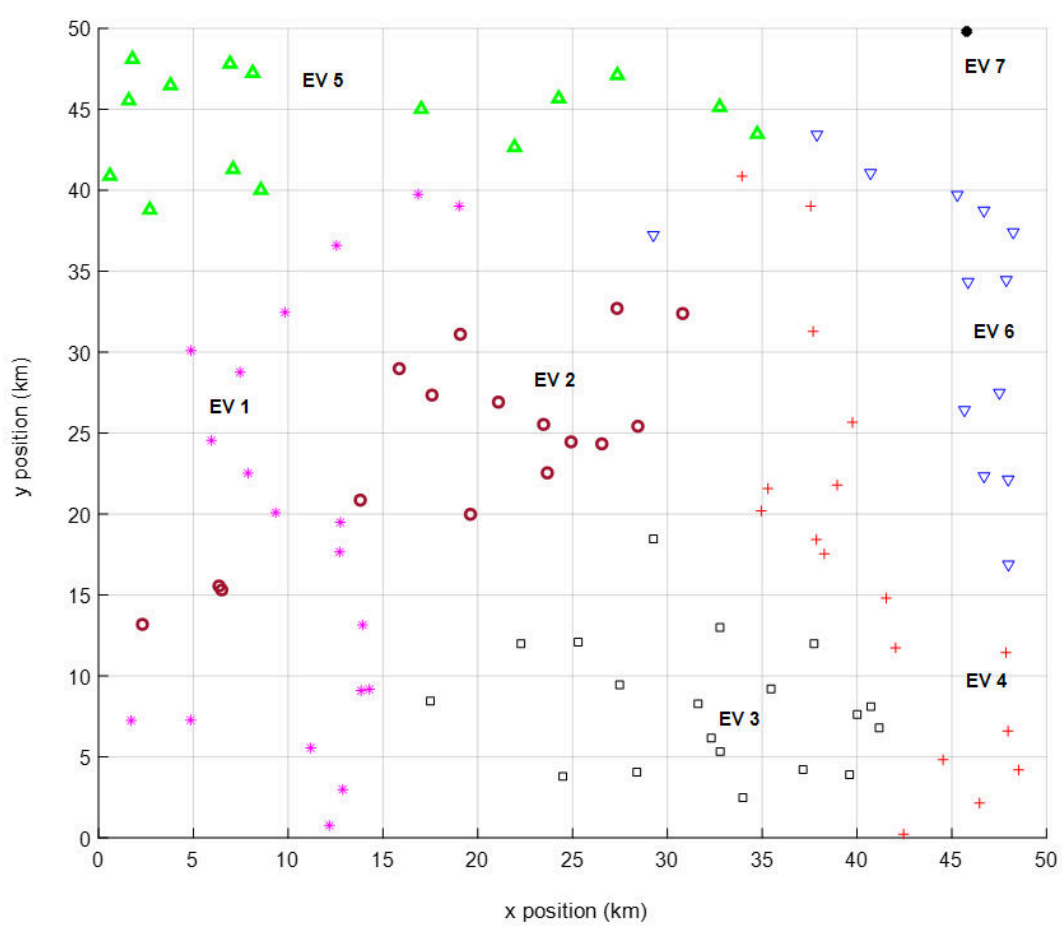

Figure 11: FMS simulation results with random initial condition 


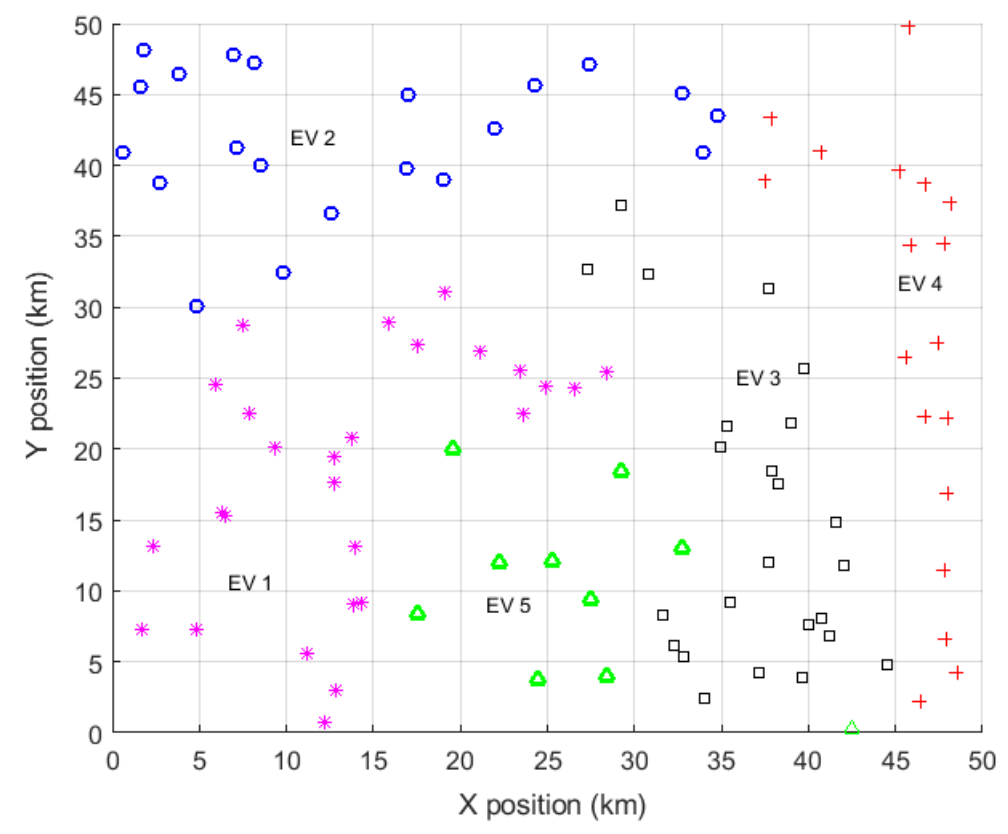

Figure 12: FMS simulation results with the best initial condition

Table 6: EVs' energy consumption in the FMS case-study using the best initial condition

\begin{tabular}{|c|c|}
\hline EV number & EV energy consumption \\
\hline 1 & $23 \mathrm{kWh}$ \\
\hline 2 & $24 \mathrm{kWh}$ \\
\hline 3 & $22 \mathrm{kWh}$ \\
\hline 4 & $23 \mathrm{kWh}$ \\
\hline 5 & $19 \mathrm{kWh}$ \\
\hline Total & $111 \mathrm{kWh}$ \\
\hline
\end{tabular}

Table 7: Overall fleet's energy consumption as a function of estimation uncertainty

\begin{tabular}{|c|c|c|c|}
\hline $\begin{array}{c}\text { Energy estimation } \\
\text { uncertainty }\end{array}$ & $\begin{array}{c}\text { Number of EVs } \\
\text { dispatched }\end{array}$ & $\begin{array}{c}\text { Overall fleet's } \\
\text { energy consumption }\end{array}$ & $\begin{array}{c}\text { Increase in overall } \\
\text { fleet energy } \\
\text { consumption }\end{array}$ \\
\hline 0 & 5 & $111.03 \mathrm{kWh}$ & $0 \%$ \\
\hline $0.5 / 24=2.08 \%$ & 6 & $113.69 \mathrm{kWh}$ & $2.39 \%$ \\
\hline $1 / 24=4.16 \%$ & 6 & $113.69 \mathrm{kWh}$ & $2.39 \%$ \\
\hline $2 / 24=8.33 \%$ & 6 & $117.38 \mathrm{kWh}$ & $5.72 \%$ \\
\hline $5 / 24=20.83 \%$ & 8 & $132.71 \mathrm{kWh}$ & $19.53 \%$ \\
\hline
\end{tabular}

\section{Conclusions}

In this study, a simulation model was developed for EV energy consumption estimation to be used in a FMS. The proposed estimator includes EV, driver and terrain models. The proposed estimator's performance was validated against field test results on specific routes. Although the proposed estimator demonstrates a very good performance (an error less than $3 \%$ in energy consumption estimation except in the case of windy and rainy test condition), its 
limitations should also be considered to recognise the improvement directions. For example, in this study only a limited number of routes were tested. However, the same approach can be applied to other routes to cover all target points in an area where FMS operates. Another limiting assumption was the driver model. According to some literature, human drivers have different driving styles and consequently, various energy consumption values are expected when they drive the same route. In this study, one driver is used to eliminate the inconsistencies and errors due to driving style variation. A good reason for this simplification is the existing trend towards driverless cars that is expected to happen in the near future. A fleet of driverless cars can operate more consistently and consequently the energy consumption estimation would be possible using the proposed approach. Regarding the implication aspects for nonautonomous vehicles, in current practice, fleet operators can continuously monitor their drivers and consequently, it would be clear which driver spends lower/higher than the average energy consumption when travelling the same route during the same time. That sort of data can be used to extract a 'modification factor' for each driver to generate a more realistic energy consumption value based on the average expected value (to be generated by the estimator).

To extend this study for a practical application, two components should be added to the estimator: (i) weather condition, and (ii) traffic condition. The weather condition including wind and rain were not considered in the proposed model. The results demonstrated that the wind can cause more drag resistant force that increases energy consumption. On the other hand, rainy weather condition caused the vehicle screen wiper to work continuously. As a result, 19\% increase in energy consumption was observed in windy and rainy weather compared to the average expected value during normal weather condition. Traffic condition was kept consistent by doing the tests always on the same day and during the same time, whereas in reality, traffic condition can change significantly. The good thing is that the traffic data is available online. So, to improve the result, online traffic data can be used in predictions by considering it as a modification factor in energy consumption estimation.

Finally, it was demonstrated how the performance of a FMS can be improved using an accurate energy estimator. This is very important to know how much the performance of a FMS is sensitive to energy consumption estimation accuracy, which is another novelty of this study. The simulation results demonstrated a case-study in which the total energy consumption of an EV fleet was improved significantly by improving the estimation accuracy that was showing how the uncertainties in EV energy consumption estimation would limit the overall performance of a FMS. 


\section{References}

Adam C., Wanielik G., 2012, Map-based Driving Profile Simulation for Energy Consumption Estimation of Electric Vehicles, $15^{\text {th }}$ International IEEE Conference on Intelligent Transportation Systems, Anchorage, AK, USA.

Bandeira J. M., Carvalho D. O., Khattak A. J., Rouphail N. M., Fontes T., Fernandes P., Pereira S. R., Coelho M. C., 2016, Empirical Assessment of Route Choice Impact on Emissions over Different Road Types, Traffic Demands, and Driving Scenarios, International Journal of Sustainable Transportation 10(3): 271-283.

Betz J., Werner D., Lienkamp M., 2016, Fleet Disposition Modeling to Maximize Utilization of Battery Electric Vehicles in Companies with On-Site Energy Generation, Transportation Research Procedia, 19: 241 - 257.

Chen G., Zhang W-G, Zhang X-N, 2013, Speed Tracking Control of a Vehicle Robot Driver System Using Multiple Sliding Surface Control Schemes, International Journal of Advanced Robotic Systems 10(2).

Cordeau J-F., Laporte G., Mercier A., 2001, A Unified Tabu Search Heuristic for Vehicle Routing Problems with Time Windows, Journal of the Operational Research Society, 52(8): 928-936.

Corti A., Ongini C., Tanelli M., Savaresi S.M., 2013, Quantitative Driving Style Estimation for Energy-oriented Applications in Road Vehicles, IEEE International Conference on Systems, Man, and Cybernetics, pp. 3710 - 3715, Manchester, UK.

Dantzig G.B., Ramser J.H., 1959, The Truck Dispatching Problem, Management Science, 6(1): 80-91.

Delgado O. F., Clark N. N., Thompson G. J, 2012, Heavy Duty Truck Fuel Consumption Prediction Based on Driving Cycle Properties, International Journal of Sustainable Transportation, 6:338-361.

Fotouhi A., Auger D. J., Cleaver T., Shateri N., Propp K., Longo S., 2016, Influence of Battery Capacity on Performance of an Electric Vehicle Fleet, IEEE International Conference on Renewable Energy Research and Applications (ICRERA), Birmingham, UK.

Giglio D., Paolucci M., Sacile R., 2004, A Dynamic Routing Strategy for the Real-Time Management of a Fleet of Tank Trucks, IEEE International Conference on Systems, Man and Cybernetics, The Hague, Netherlands, 7:6250-6255.

Giménez-Gaydou D. A., Ribeiro A. S. N., Gutiérrez J., Pais Antunes A., 2016, Optimal Location of Battery Electric Vehicle Charging Stations in Urban Areas: A New Approach, International Journal of Sustainable Transportation, 10(5): 393-405.

Graser A., Asamer J., Ponweiser W., 2015, The Elevation Factor: Digital Elevation Model Quality and Sampling Impacts on Electric Vehicle Energy Estimation Errors, IEEE, International Conference on Models and Technologies for Intelligent Transportation Systems (MT-ITS), Budapest, Hungary. 
Guzzella L., Sciarretta A., 2013, Vehicle Propulsion Systems, Introduction to Modelling and Optimization, Springer.

Hayes J. G., de Oliveira R. P. R., Vaughan S., Egan M. G., 2011, Simplified Electric Vehicle Power Train Models and Range Estimation, IEEE Vehicle Power and Propulsion Conference, Chicago, IL, pp. 1-5

Hu J., Morais H., Sousa T., Lind M., 2016, Electric Vehicle Feet Management in Smart Grids: A Review of Services, Optimization and Control Aspects, Renewable and Sustainable Energy Reviews, 56: 1207-1226.

Jayakumar A., Ingrosso F., Rizzoni G., Meyer J., Doering J., 2014, Crowd Sourced Energy Estimation in Connected Vehicles, IEEE International Electric Vehicle Conference (IEVC), Florence, Italy.

Jeong I. J., Illades Boy C. A., 2018, Routing and refueling plans to minimize travel time in alternative-fuel vehicles, International Journal of Sustainable Transportation 12(8): 583-591

Felipe J., Amarillo J. C., Naranjo J. E., Serradilla F., Diaz A., Energy consumption estimation in electric vehicles considering driving style, IEEE 18th International Conference on Intelligent Transportation Systems, 2015, Las Palmas, Spain.

Kichun Jo, Jaesung Chung, Myoungho Sunwoo, 2013, 3D Map Generation Algorithm for Energy Management System on Electric Vehicles, World Electric Vehicle Symposium and Exhibition (EVS27), Barcelona, Spain.

Kirkpatrick S., Gelatt Jr. C. D., Vecchi M. P., 2007, Optimisation by Simulated Annealing, Science, New Series, 220 (4598): 671-680.

Maalej K., Kelouwani S., Agbossou K., Dubé Y., Henao N. F., 2015, Long-Trip Optimal Energy Planning With Online Mass Estimation for Battery Electric Vehicles, IEEE Transactions on Vehicular Technology, 64 (11): 4929-4941.

Qiang Song, Lin Luo, 2014, Speed-Tracking Driver Model Used in Hardware-in-Loop Simulation, Applied Mechanics and Materials 447: 1222-1226.

Shen Lin, 1965, Computer Solutions of the Traveling Salesman Problem, Bell System Technical Journal, 44(10): 2245-2269.

Trigui O., Mejri E., Dubé Y., Kelouwani S., Agbossou K., 2017, Energy Efficient Routing Estimation in Electric Vehicle with Online Rolling Resistance Estimation, IEEE Vehicle Power and Propulsion Conference, Belfort, France.

Trigui O., Dubé Y., Kelouwani S., Agbossou K., 2016, Comparative estimation of electric vehicle rolling resistance coefficient in winter conditions, IEEE Vehicle Power and Propulsion Conference, 2016, Hangzhou, China.

Vatanparvar K., Faezi S., Burago I., Levorato M., Abdullah Al Faruque M., 2018, Extended Range Electric Vehicle with Driving Behavior Estimation in Energy Management, IEEE Transactions on Smart Grid, DOI 10.1109/TSG.2018.2815689. 
Venkata Narasimha K.S., Kumar M., 2011 Ant Colony Optimization Technique to Solve the Min-Max Single Depot Vehicle Routing Problem, American Control Conference, San Francisco, USA.

Zhu Chao, Chen Xiaohong, 2013, Optimizing Battery Electric Bus Transit Vehicle Scheduling with Battery Exchanging: Model and Case Study, Procedia - Social and Behavioral Sciences, 96: 2725-2736.

Ziegler J.G., Nichols N.B., 1942, Optimum Settings for Automatic Controllers, Transactions of the ASME, 64: 759-768. 
$2019-10-30$

\section{Electric vehicle energy consumption estimation for a fleet management system}

\section{Fotouhi, Abbas}

Taylor and Francis

Fotouhi A, Shateri N, Laila DS, Auger DJ. (2020) Electric vehicle energy consumption estimation for a fleet management system. International Journal of Sustainable Transportation, Volume 15, Issue 1, 2020. pp. 40-54 https://doi.org/10.1080/15568318.2019.1681565

Downloaded from Cranfield Library Services E-Repository 